\title{
Role of MRI in Differentiating Various Posterior Cranial Fossa Space-Occupying Lesions Using Sensitivity and Specificity: A Prospective Study
}

\author{
Prabhakaran Tamilchelvan ${ }^{1}$, Deb K. Boruah ${ }^{1,2}$, Bidyut B. Gogoi ${ }^{3}$, Rudrakanta Gogoi ${ }^{1}$ \\ 1. Radiodiagnosis, Assam Medical College and Hospital, Dibrugarh, IND 2. Radiodiagnosis, Tezpur Medical College, \\ Tezpur, IND 3. Pathology, Assam Medical College and Hospital, Dibrugarh, IND
}

Corresponding author: Deb K. Boruah, debpal80@gmail.com

\begin{abstract}
Background: Any abnormal space-occupying posterior fossa lesion may directly involve the vital structures like the brain stem, cranial nerves, cerebellum, vertebrobasilar artery, and venous sinuses, which makes the surgical approach and total excision very difficult. Hence for these reasons, precise evaluation of posterior fossa lesion with MRI is a must to visualize the vital structures, which helps in planning and safe surgery.
\end{abstract}

Objective: This study aimed to evaluate the added value of diffusion-weighted imaging and magnetic resonance spectroscopy in the localization, extension, characterization, differentiation of various posterior fossa space-occupying lesions, and correlating with the histopathological result.

\begin{abstract}
Materials and methods: This prospective study comprised of 40 patients who were suspected with posterior fossa space-occupying lesions on basis of clinical features or on CT scan. All patients were evaluated using conventional as well as newer MRI techniques using Siemens 1.5 Tesla MRI scanner (Siemens Medical System, Erlangen, Germany). Diffusion-weighted imaging (DWI) was done in all patients and magnetic resonance spectroscopy (MRS) was done in 27 patients. Based on the MRI findings, various posterior fossa lesions were classified as neoplastic or non-neoplastic. The neoplastic lesions were further classified as benign and malignant. The MRI findings were correlated with histopathological findings or follow-up.
\end{abstract}

Statistical analysis: Independent sample t-test was used to compare the mean apparent diffusion coefficient (ADC) values of various posterior fossa space-occupying lesions. Receiver operating characteristic (ROC) curve analysis was done to determine the optimal cut-off mean ADC values and choline/creatinine (Cho/cr) ratios for various benign and malignant posterior fossa tumors.

Review began 06/21/2021 Review ended 06/27/2021 Published 07/12/2021

๑) Copyright 2021

Tamilchelvan et al. This is an open access article distributed under the terms of the Creative Commons Attribution License CC-BY 4.0., which permits unrestricted use, distribution, and reproduction in any medium, provided the original author and source are credited.
Results: Of 40 patients with posterior fossa lesions, 23 were males and 17 were females with a mean age of $34.67 \pm 1.93[\mathrm{SD}]$ years. Metastases were the most common posterior fossa lesions in our study sample and found in seven patients (17.5\%) followed by vestibular schwannomas and brainstem gliomas in five patients (12.5\%) each, demyelinating lesion in four patients (10\%), tubercular abscess in three patients (7.5\%), hemangioblastoma, tuberculoma, arachnoid cyst, epidermoid cyst, pilocytic astrocytoma, low-grade glioma in two patients (5\%) each, meningioma, medulloblastoma, pyogenic abscess and high-grade glioma in one patient (2.5\%) each. The mean ADC value of benign tumors was higher than that of malignant tumors and this difference was found to be significant $(\mathrm{p}=0.019)$. The cut-off ADC value $1.022 \times 10^{-3} \mathrm{~mm}^{2} / \mathrm{s}$ had a sensitivity of $78.6 \%$ and specificity of $66.7 \%$. MRS played important role in differentiating neoplastic from non-neoplastic lesions and benign from malignant tumors. The cut-off Cho/cr ratio of 1.25 had a sensitivity of $66.7 \%$, specificity of $85.7 \%$ to differentiate benign from malignant tumors.

Conclusion: Conventional MRI sequences able to diagnose most of the benign-appearing lesions of posterior fossa, however, adding advanced MRI sequences like diffusion-weighted imaging and MR spectroscopy helps us to differentiate and diagnose various posterior fossa lesions even closer to the actual histopathological diagnosis.

Categories: Neurology, Radiology, Neurosurgery

Keywords: magnetic resonance imaging (mri), diffusion-weighted imaging (dwi), magnetic resonance spectroscopy (mrs), tumor, posterior fossa

\section{Introduction}

The posterior fossa of the brain consists of vital structures like the brainstem, cerebellum, lower cranial nerves, vertebrobasilar arteries, and venous sinuses. The posterior fossa is the spaces above the foramen of the magnum and below the tentorial cerebelli [1]. Computed tomography of the posterior fossa is limited by the beam hardening artifacts and which adversely hamper the quality of images. MRI has played an increasingly important role and is the investigation of choice in patients suspected of harboring lesions in the posterior fossa. T1-weighted images provide excellent anatomical details while T2-weighted images 
provide pathological information [2].

Conventional MR imaging provides diagnosis with a success rate of 30-90\%. However, advanced MRI sequences like diffusion-weighted imaging (DWI), MR perfusion, and magnetic resonance spectroscopy (MRS) can provide immense value in the characterization and diagnosis of various posterior fossa spaceoccupying lesions $[3,4]$.

DWI is helpful in distinguishing between the brain abscesses, necrotic, and cystic neoplasms on MRI. Increased tumor cellularity in neoplasm causes a relative reduction in the apparent diffusion coefficient (ADC) values and that helps in differentiation between the medulloblastoma, ependymoma, and pilocytic astrocytoma [3]. By adding DWI and MR spectroscopy over conventional MRI can negate the need for biopsy in various posterior fossa tumors [3]. DWI is an excellent tool used in differentiating epidermoid from arachnoid cysts even difficult to differentiate with conventional MR sequences [4].

MRS gives information related to cell membrane proliferation, neuronal damage, energy metabolism, and necrotic transformation of brain or tumor tissues. MRS gives information at the molecular level rather than structural and anatomic imaging of tissues and organs by identifying various chemical metabolites [5,6]. MR spectroscopy can differentiate between the non-neoplastic and neoplastic lesions, benign and malignant brain lesions $[6,7]$.

This study aimed to evaluate the added value of diffusion-weighted imaging and MRS in the localization, extension, characterization, differentiation of various posterior fossa space-occupying lesions, and correlating with the histopathological result.

\section{Materials And Methods}

\section{Study design}

After approval from the institutional ethics review committee, a hospital-based cross-sectional prospective study was conducted. This study group comprised of 40 patients with CT proven or clinical suspicion of posterior fossa space-occupying lesion presented to the Department of Radiodiagnosis in a tertiary care center of Northeast India from July 2017 to June 2018. Informed consent was obtained from the patient/parents/guardian before undergoing an MRI scan (Figure 1). Patients with posterior fossa stroke, patients with a history of head trauma, patients with congenital posterior fossa malformation like DandyWalker cyst, inadequate or noisy MR spectroscopy, and lesion size less than $10 \mathrm{~mm}$ were excluded from the study.

\section{Flow chart of Study design}

\section{Potentially eligible participants of posterior \\ fossa space occupying lesions $(n=63)$}

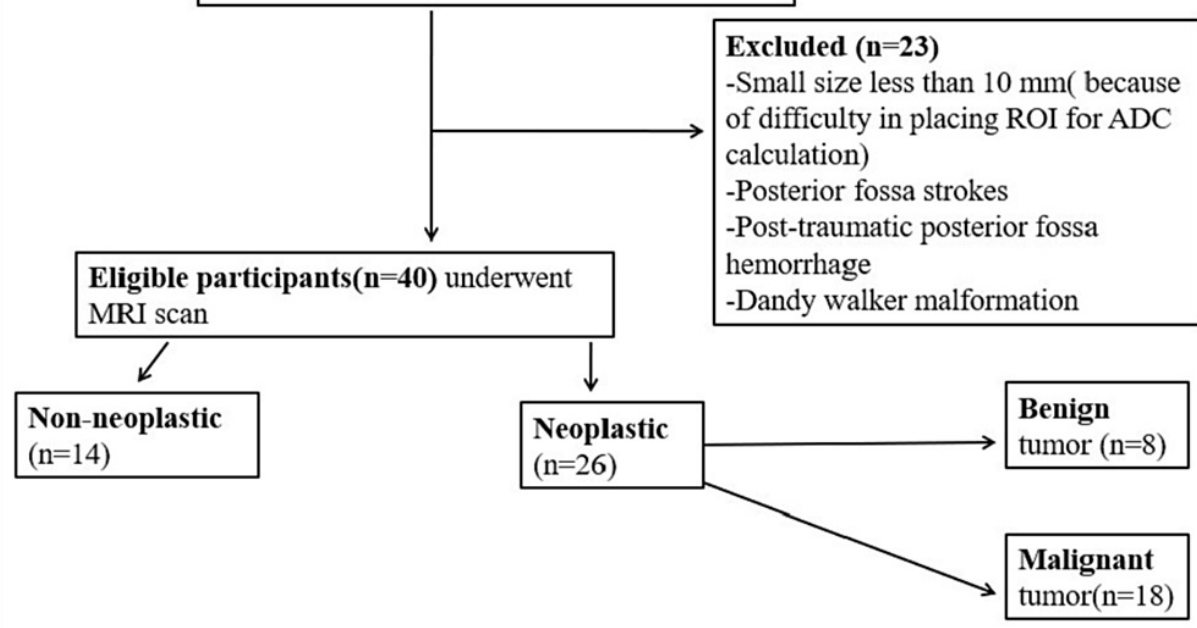

FIGURE 1: Flow chart of the study design.

$\mathrm{ROI}$ : region of interest; ADC: apparent diffusion coefficient

\section{Test method}


All patients were subjected to an MRI scan of the brain in a supine position in Siemens Magnetom Avanto 1.5 Tesla MR scanner (Siemens Medical System, Erlangen, Germany). Conventional MR sequences like axial T1WI, T2WI, fluid-attenuated inversion recovery (FLAIR), susceptibility-weighted image (SWI), diffusionweighted image (DWI), sagittal T1W1, and coronal T2WI sequences were obtained using 4-5 mm slice thickness. DWI using echo-planar imaging (EPI) was done in all the patients before contrast administration. Post gadolinium fat-suppressed T1W images were obtained in different planes. The MR spectroscopy data were obtained in 27 patients only. Both single and multi-voxel techniques (chemical shift imaging $\{\mathrm{CSI}\}$ sequence) were employed in our study with TE $135 \mathrm{msec}$ and $30 \mathrm{msec}$. Both stimulated echo acquisition mode (STEAM) and point resolved spectroscopy (PRESS) techniques were employed. Various MRI sequences are shown in (Table 1).

\begin{tabular}{|c|c|c|c|c|c|c|c|c|}
\hline Parameters & T2W & FLAIR & T1W & $\begin{array}{l}\text { Coronal } \\
\text { T2W }\end{array}$ & $\begin{array}{l}\text { Sagittal } \\
\text { T1W }\end{array}$ & DWI & SWI & $\begin{array}{l}\text { Post-gadolinium fat- } \\
\text { suppressed T1W }\end{array}$ \\
\hline Imaging plane & axial & axial & axial & coronal & Sagittal & axial & axial & axial, coronal \& sagittal \\
\hline TE/TR (msec) & $100 / 3500$ & $\begin{array}{l}90 / 3500 \\
(\mathrm{TI}=2500)\end{array}$ & $\begin{array}{l}7- \\
9 / 500\end{array}$ & $90 / 3600$ & $\begin{array}{l}90 / 3600 \\
\mathrm{Tl}=160\end{array}$ & $90 / 3900$ & 200/1500 & $10 / 550$ \\
\hline FOV (cm) & $190-200$ & $190-200$ & $\begin{array}{l}190- \\
240\end{array}$ & $200-240$ & $190-200$ & $\begin{array}{l}190- \\
200\end{array}$ & $190-200$ & $190-200$ \\
\hline $\begin{array}{l}\text { Slice thickness } \\
(\mathrm{mm})\end{array}$ & 4 & 4 & 5 & 4 & 4 & 4 & 3 & 3.5 \\
\hline $\begin{array}{l}\text { Interslice gap } \\
\text { (mm) }\end{array}$ & 0.5 & 0.5 & 1.5 & 1 & 0.3 & 0.4 & 1.5 & 0.3 \\
\hline No of slices & 30 & 30 & 21 & 25 & 25 & 30 & 120 & 30 \\
\hline Matrix & $\begin{array}{l}272 \times \\
215\end{array}$ & $212 \times 186$ & $\begin{array}{l}234 \times \\
384\end{array}$ & $234 \times 384$ & $252 \times 216$ & $80 \times 79$ & $\begin{array}{l}220 x \\
220\end{array}$ & $256 \times 256$ \\
\hline Flip angle & $90^{0}$ & $90^{0}$ & $90^{\circ}$ & $90^{0}$ & $90^{\circ}$ & $90^{0}$ & $90^{\circ}$ & $90^{\circ}$ \\
\hline $\begin{array}{l}\text { Echo train } \\
\text { length }\end{array}$ & 19 & 20 & 13 & 20 & 22 & - & 94 & 4 \\
\hline b-value $\left(\mathrm{s} / \mathrm{mm}^{2}\right)$ & - & - & & - & - & 1000 & - & - \\
\hline
\end{tabular}

\section{TABLE 1: MR imaging sequences of the brain in posterior fossa lesions.}

FS: fat-suppressed; TE: time of echo; TR: time of relaxation; FLAIR: fluid-attenuated inversion recovery; DWI: diffusion-weighted image; SWI: susceptibility-weighted image

\section{Analysis}

Two radiologists, one having experience of neuroradiology for 13 years and another having four years of experience prospectively analyzed the MRI images. The two radiologists were blinded to the clinical history or previous radiological reports of the patients. The size of the posterior fossa space-occupying lesion and $A D C$ values were calculated independently by the two radiologists and mean ADC values were used for the results. The MR spectroscopy parameters especially the choline, N-acetyl aspartate (NAA), lipid, lactate, aminoacid peaks, and choline/creatinine ratio were analyzed independently.

Analysis of Conventional MR Images

We evaluated the following characteristics of a space-occupying lesion in the posterior fossa like lesion sizes, locations, margins, T1WI and T2WI appearances, perilesional edema, and lesion heterogeneity. The posterior fossa lesion size was obtained from the largest dimension of a lesion. The location of a posterior fossa lesion is identified according to the involvement of posterior fossa structures. Margins of spaceoccupying lesion in posterior fossa classified into well-defined and ill-defined. The "well-defined" margin was considered when the margin of space-occupying lesion was differentiated from surrounding structures. An "ill-defined" lesion was considered when the margin of a lesion was ill-defined. Lesion heterogeneity was considered when there were mixed-signal intensities on T1WI or T2W images. 
Minimum, maximum, and mean ADC values of a posterior fossa space-occupying lesion were calculated from placing either round or elliptical region of interests (ROIs) over the solid, enhancing, non-necrotic, and/or DWI restricted part of a solid enhancing lesion. In a peripherally enhancing cystic lesion, ROIs were placed in the enhancing wall of the lesions. In the case of cystic lesions and enhancing mural nodules, ROIs were placed in the enhancing mural nodule. ADC values were measured in the operating system console using multiple uniform sizes (area: minimum $10 \mathrm{~mm}^{2}$, maximum $50 \mathrm{~mm}^{2}$ ). We divided the lesion into four quadrants and calculated the ADC values from four quadrants and the final mean ADC value was calculated. In patients with multiple posterior fossa lesions, the largest lesion was selected for calculation of the mean ADC value.

\section{MR Spectroscopy Data Analysis}

Appropriate-sized voxels were chosen to analyze the metabolites with MR spectroscopy. MR spectroscopy spectra were analyzed for various metabolites like choline, creatinine, $\mathrm{N}$-acetyl aspartate, lactate, lipid, alanine, and amino acids. Metabolite ratios like Cho/cr were generated. Mean values of the Cho/cr ratio of different lesions were calculated and compared with a final diagnosis. Mean Cho/cr ratio of neoplastic and non-neoplastic lesions, benign and malignant tumors were then compared and their statistical significance was calculated.

Histopathological Analysis/Final Diagnosis

The final diagnosis was achieved by typical MR imaging findings in $n=15$ patients, histopathological diagnosis of posterior fossa space-occupying lesion was established on surgically resected specimens in $n=19$ patients, and follow-up post-treatment scan in $n=6$ patients.

Statistical Analysis

All the statistical analysis was performed using Statistical Package for Social Sciences (SPSS) version 16 (Armonk, NY: IBM Corp.). The mean ADC values of various lesions were calculated by an independent sample t-test. The optimal cut-off mean $\mathrm{ADC}$ values and $\mathrm{Cho/cr}$ ratio for various posterior fossa tumors were obtained from receiver operating characteristic (ROC) curve analysis.

\section{Results}

Of 40 patients with posterior fossa lesions, 23 were males and 17 were females with a mean age of $34.67 \pm 1.93[\mathrm{SD}]$ years. Maximum nine patients (22.5\%) each were in the age group of 11-20 years and 31-40 years followed by seven patients (17.5\%) in the age group of $41-50$ years and five patients (12.5\%) in the age group of 21-30 years. Only seven patients (17.5\%) were above the age of 50 years. Headache and vomiting were the most common presenting symptoms found in 24 patients $(60 \%)$.

Thirty patients (75\%) had intra-axial space-occupying lesions while 10 patients (25\%) had extra-axial lesions. In the intra-axial compartment, the cerebellum is the most commonly involved in 19 patients (47.5\%) followed by brain stem in six (15\%) patients. In the extra-axial compartment, cerebello-pontine (CP) angle cistern was most commonly involved in five patients (12.5\%).

Metastases were the most common posterior fossa lesions in our study sample and found in seven patients (17.5\%) followed by vestibular schwannomas and brainstem gliomas in five patients (12.5\%) each, demyelinating lesion in four patients (10\%), tubercular abscess in three patients (7.5\%), hemangioblastoma, tuberculoma, arachnoid cyst, epidermoid cyst, pilocytic astrocytoma, low-grade glioma in two patients (5\%) each, meningioma, medulloblastoma, pyogenic abscess and high-grade glioma in one patient (2.5\%) each. The different posterior fossa lesions with their mean ADC values are shown in Table 2. 


\section{Cureus}

\begin{tabular}{|c|c|c|}
\hline Posterior fossa lesion & Number/percentage of cases & Mean ADC value $\times 10^{-3} \mathrm{~mm}^{2} / \mathrm{s}$ \\
\hline Metastases & $7(17.5 \%)$ & $0.886 \pm 0.21$ \\
\hline Brainstem glioma & $5(12.5 \%)$ & $1.093 \pm 0.36$ \\
\hline Vestibular schwannoma & $5(12.5 \%)$ & $1.121 \pm 0.41$ \\
\hline Demyelinating lesions & $4(10 \%)$ & $0.893 \pm 0.02$ \\
\hline Tubercular abscess & $3(7.5 \%)$ & $0.697 \pm 0.14$ \\
\hline Hemangioblastoma & $2(5 \%)$ & $1.604 \pm 0.24$ \\
\hline Tuberculoma & $2(5 \%)$ & $1.019 \pm 0.08$ \\
\hline Arachnoid cyst & $2(5 \%)$ & $3.095 \pm 0.10$ \\
\hline Epidermoid cyst & $2(5 \%)$ & $0.714 \pm 0.11$ \\
\hline Pilocytic astrocytoma & $2(5 \%)$ & $1.615 \pm 0.03$ \\
\hline Low-grade glioma & $2(5 \%)$ & $1.232 \pm 0.27$ \\
\hline Meningioma & $1(2.5 \%)$ & 0.843 \\
\hline Medulloblastoma & $1(2.5 \%)$ & 0.680 \\
\hline Pyogenic abscess & $1(2.5 \%)$ & 0.384 \\
\hline High-grade cerebellar glioma & $1(2.5 \%)$ & 0.708 \\
\hline Benign tumor & $8(20 \%)$ & $1.279 \pm 0.38$ \\
\hline Malignant tumor & $18(45 \%))$ & $0.954 \pm 0.27$ \\
\hline
\end{tabular}

TABLE 2: Various posterior fossa space-occupying lesions with their mean ADC values in $\mathbf{4 0}$ patients.

ADC: apparent diffusion coefficient

Posterior fossa metastasis was the most common intra-axial lesion in posterior fossa lesion (Figures $2 A-2 F$ ) while vestibular schwannoma was the most common extra-axial tumor (Figures 3A-3F). 


\section{Cureus}
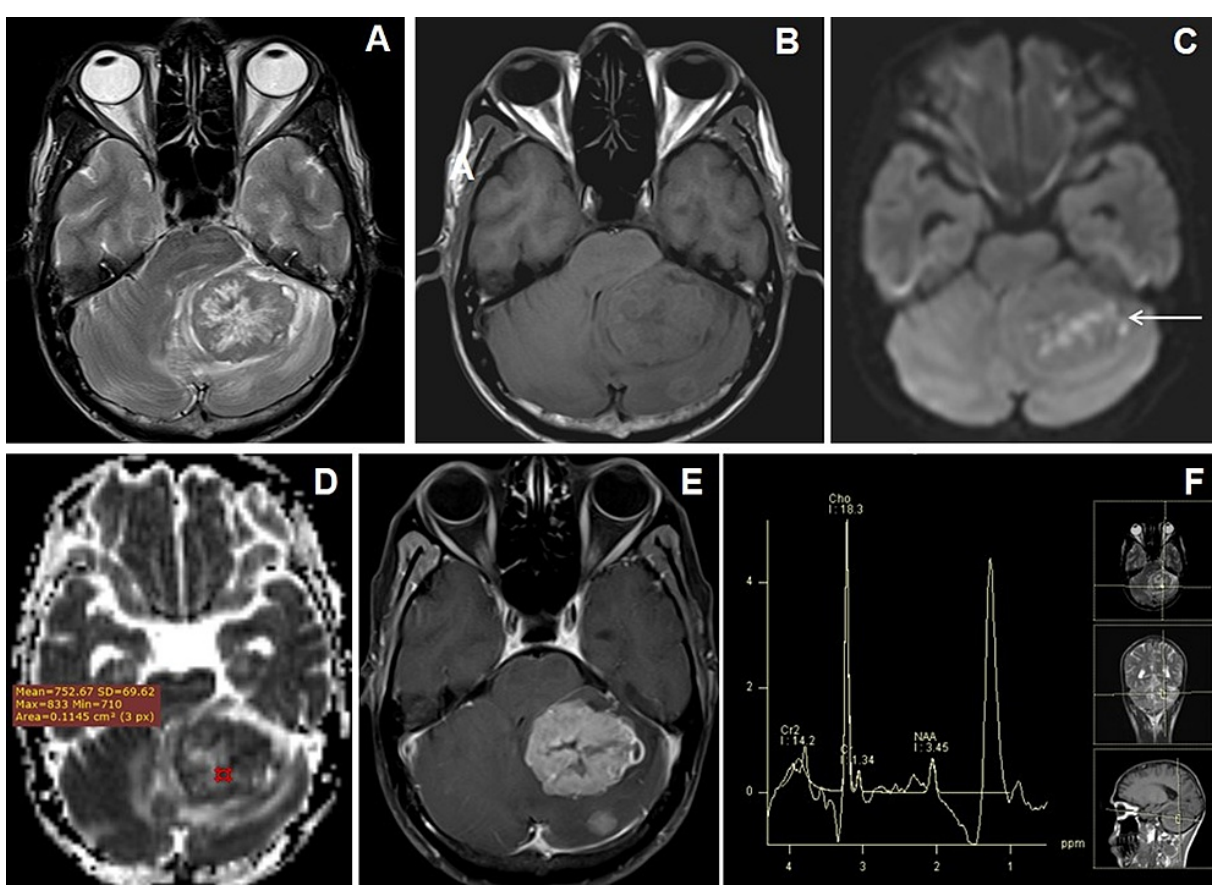

FIGURE 2: Cranial MRI of a 49 years male who presented with seizures and headache with cerebellar metastasis from adenocarcinoma of lung.

(A) Axial T2WI image shows central T2W hyperintense and peripheral isointense larger lesion in the left cerebellar hemisphere with perifocal edema. (B) Axial T1WI image shows isointense signal intensities within the larger lesion. Another smaller isointense nodular lesion was seen in the left cerebellar hemisphere posterior to the larger one. (C and D) Axial DWI and ADC map images show patchy diffusion restriction ( $\leftarrow$ arrow) with low to variable ADC values. (E) Axial T1W post-contrast image shows heterogeneous enhancement of the larger lesion with central necrosis along with another solid enhancing nodule in the left cerebellum posterior to the larger one. (F) MR spectroscopy images at TE-135 ms show increased choline and lipid-lactate peaks with reduction of NAA peak.

NAA: N-acetyl aspartate; DWI: diffusion-weighted imaging; ADC: apparent diffusion coefficient 


\section{Cureus}
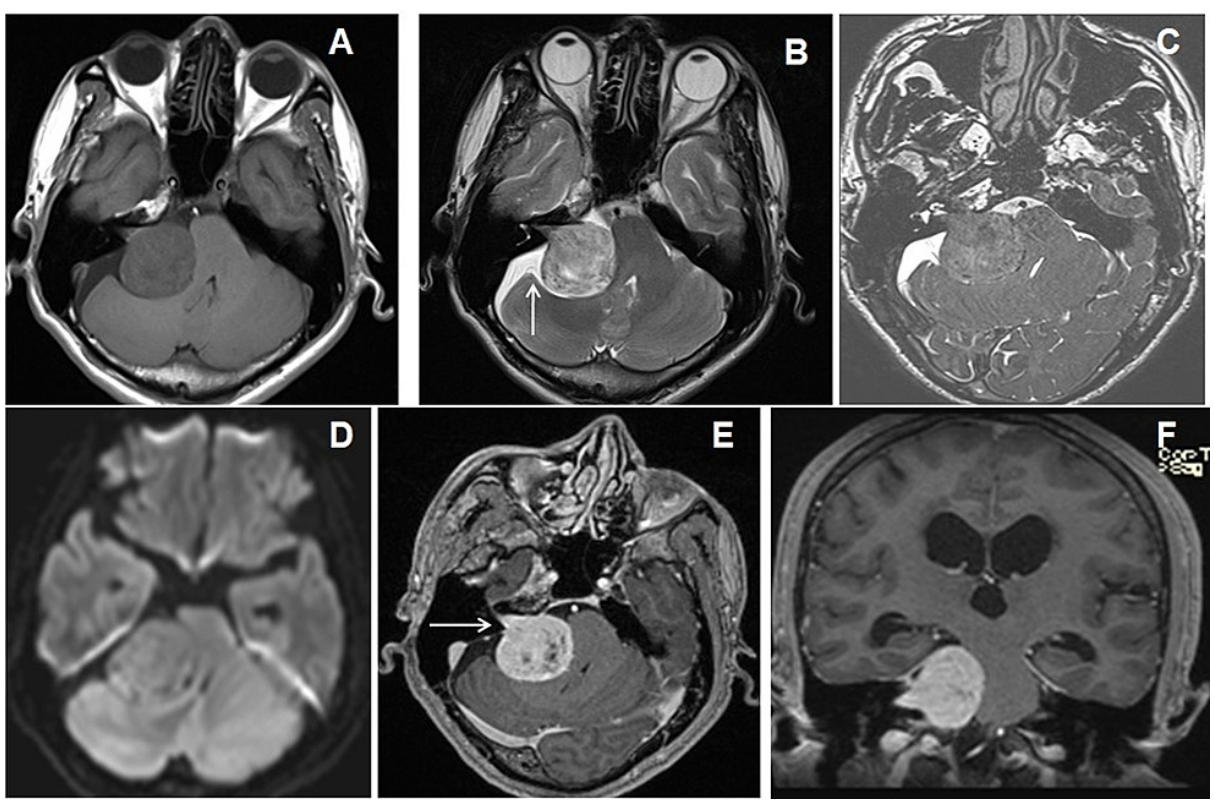

FIGURE 3: Cranial MRI of a 50-year-old male patient with vertigo and ataxia due to right-sided vestibular schwannoma.

(A and B) Axial T1WI and T2WI images show an extra-axial T1W isointense and T2 mixed signal intensity extra-axial lesion in right C-P angle cistern with CSF cleft ( $\uparrow$ arrow). (C) 3D-CISS image shows the tumoral extension into the right IAC. (D) DWI image does not show diffusion restriction. (E and F) Axial and coronal T1W post-contrast images show moderate homogenous enhancement of the lesion ( $\rightarrow$ arrow) with fine nonenhancing cystic spaces.

CSF: cerebrospinal fluid; 3D-CISS: three-dimensional constructive interference in steady state; DWI: diffusion-weighted image

DWI with ADC values clearly differentiating arachnoid cyst from the epidermoid cyst (Figures $4 A-4 F$ ). 


\section{Cureus}
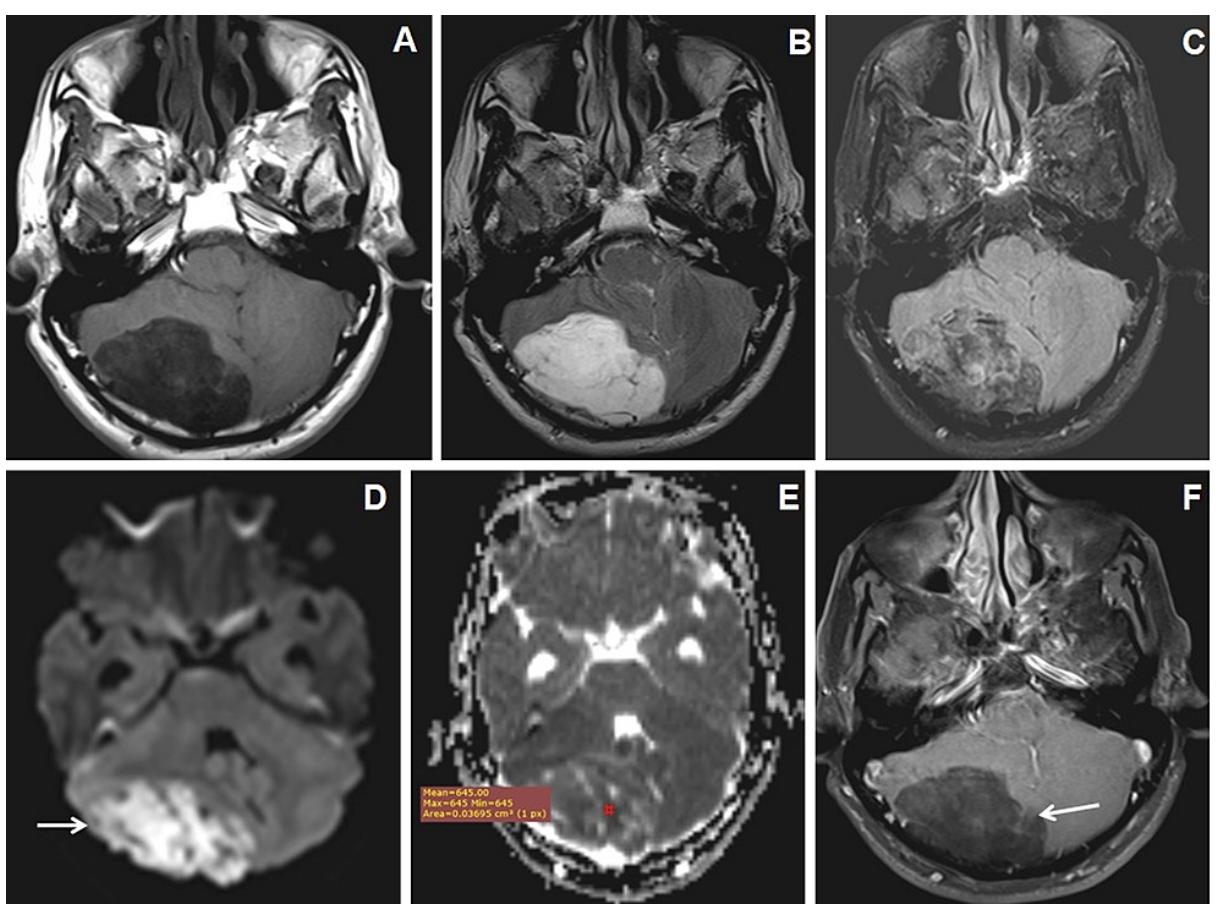

FIGURE 4: Cranial MRI of a 41-year-old male patient with headache due to epidermoid cyst.

(A and B) Axial T1WI and T2WI images show an extra-axial heterogeneous appearing T1WI hypointense and T2WI hyperintense lesion over the right cerebellar convexity with intervening T2W hypointense septae. (C) FLAIR image shows incomplete suppression of the cyst. (D and E) Axial DWI and ADC map images show restricted diffusion ( $\rightarrow$ arrow) with variable ADC values. (F) Axial T1W post-contrast image shows no enhancement $(\leftarrow$ arrow)

FLAIR: fluid-attenuated inversion recovery; DWI: diffusion-weighted imaging; ADC: apparent diffusion coefficient

DWI and MR spectroscopy differentiate tubercular (Figures $5 A-5 F$ ) and pyogenic abscess (Figures $6 A-6 F)$. Pyogenic abscess shows an increased amino acid peak at $0.9 \mathrm{ppm}$, lipid-lactate peak at $1.3 \mathrm{ppm}$, and succinate peak at $2.3 \mathrm{ppm}$ (Figure 6) on MR spectroscopy while tubercular abscesses show increased lipid peak at $0.9 \mathrm{ppm}$ with or without lactate peak at 0.9-1.33 ppm (Figure 5). 


\section{Cureus}
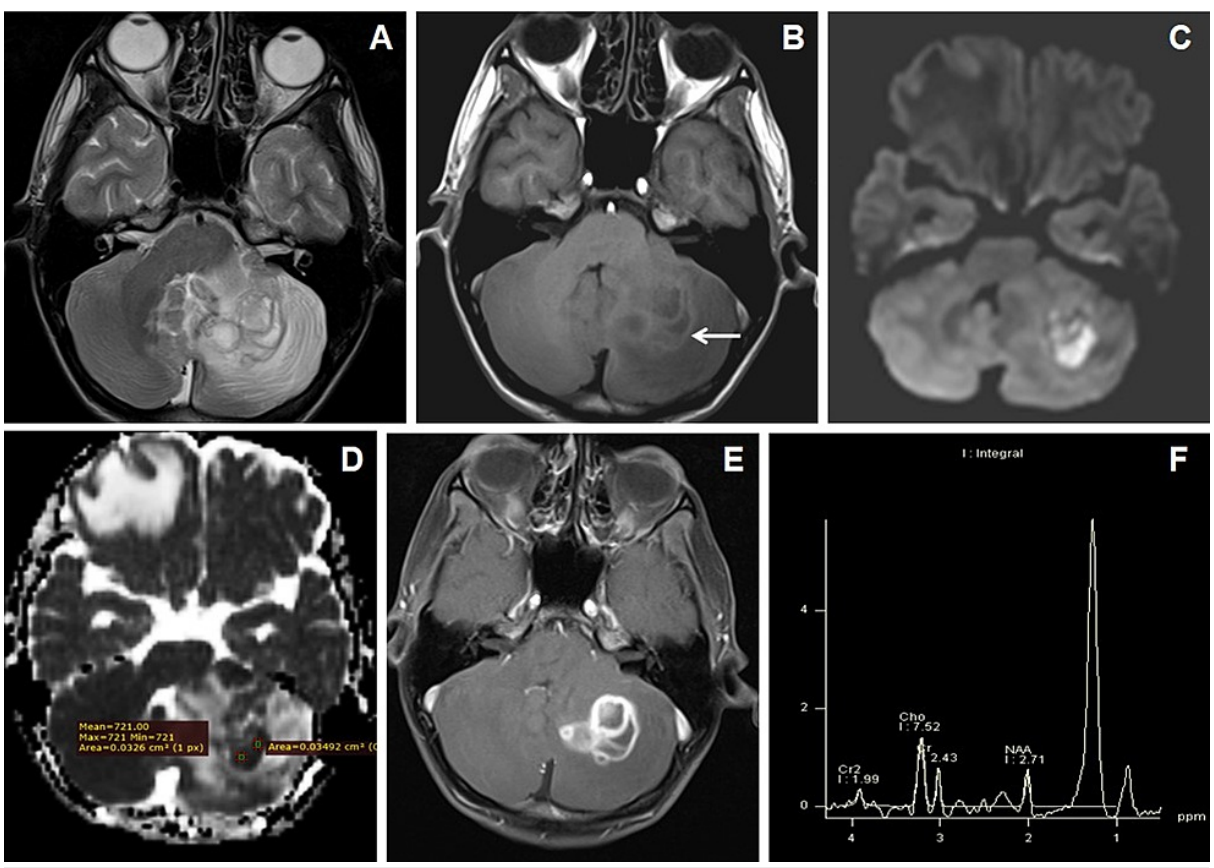

FIGURE 5: Cranial MRI of a 16-year-old male with headache and seizures due to left cerebellar tubercular abscess in a known case of pulmonary TB.

(A and B) Axial T2WI and T1WI images show conglomerated central T2W hyperintense and peripheral hypointense lesions in the left cerebellar hemisphere with mild-to-moderate perifocal edema. T1WI shows peripheral T1WI hyperintensities within the lesions ( $\leftarrow$ arrow). (C and D) Axial DWI and ADC map images show central restricted diffusion with low ADC values. (E) Axial T1W post-contrast image show thickwalled peripherally enhancing conglomerated lesions. (F) MR spectroscopy image at TE-135 ms shows a large lipid peak at 0.9-1.3 ppm and a reduction of NAA peak.

NAA: N-acetyl aspartate; DWI: diffusion-weighted imaging; ADC: apparent diffusion coefficient; TB: tuberculosis 


\section{Cureus}
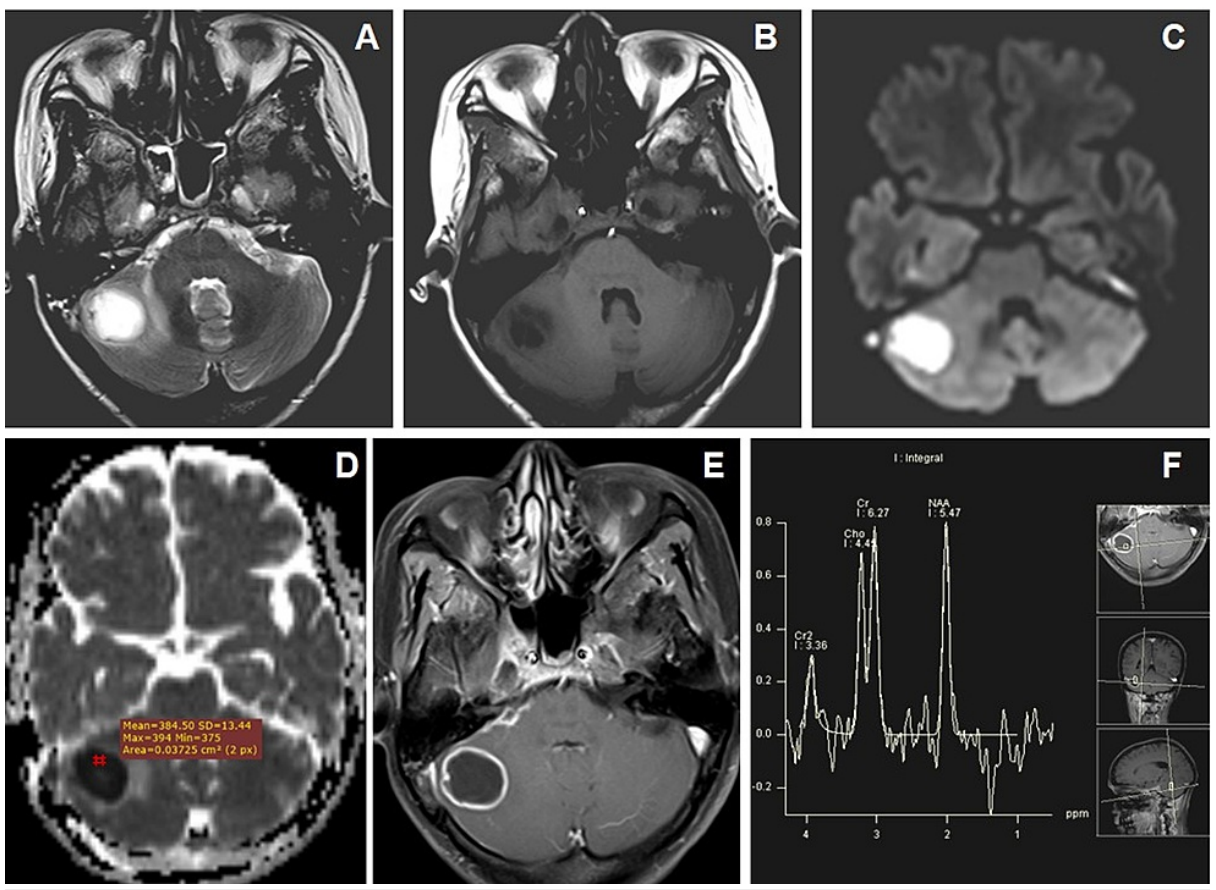

FIGURE 6: Cranial MRI of a 40-year-old female patient with headache and vomiting with and right-sided ear discharge due to pyogenic abscess in right cerebellum.

(A and B) Axial T2WI and T1WI images show central T2W hyperintense with peripheral hypointense wall lesion in the right cerebellum inciting mild perilesional edema. (C and D) Axial DWI and ADC map images show central diffusion-restriction with a low ADC value. (E) Axial T1W post-contrast image shows thickwalled peripheral rim-like enhancement of the lesion with central liquefaction. (F) MR spectroscopy image at TE-135 ms shows an amino acid peak at $0.9 \mathrm{ppm}$, a lipid-lactate peak at $1.3 \mathrm{ppm}$, and a doublet succinate peak at $2.4 \mathrm{ppm}$.

DWI: diffusion-weighted imaging; ADC: apparent diffusion coefficient

Brainstem gliomas showed diffusion restriction with low ADC values with a raised choline, lactate peaks, and Cho/cr ratio on MR spectroscopy (Figures 7A-7F). Two patients each having cerebellar pilocytic astrocytomas and hemangioblastoma showed minimally raised choline peak with a low Cho/cr ratio (less than 1.2) in MR spectroscopy. 


\section{Cureus}
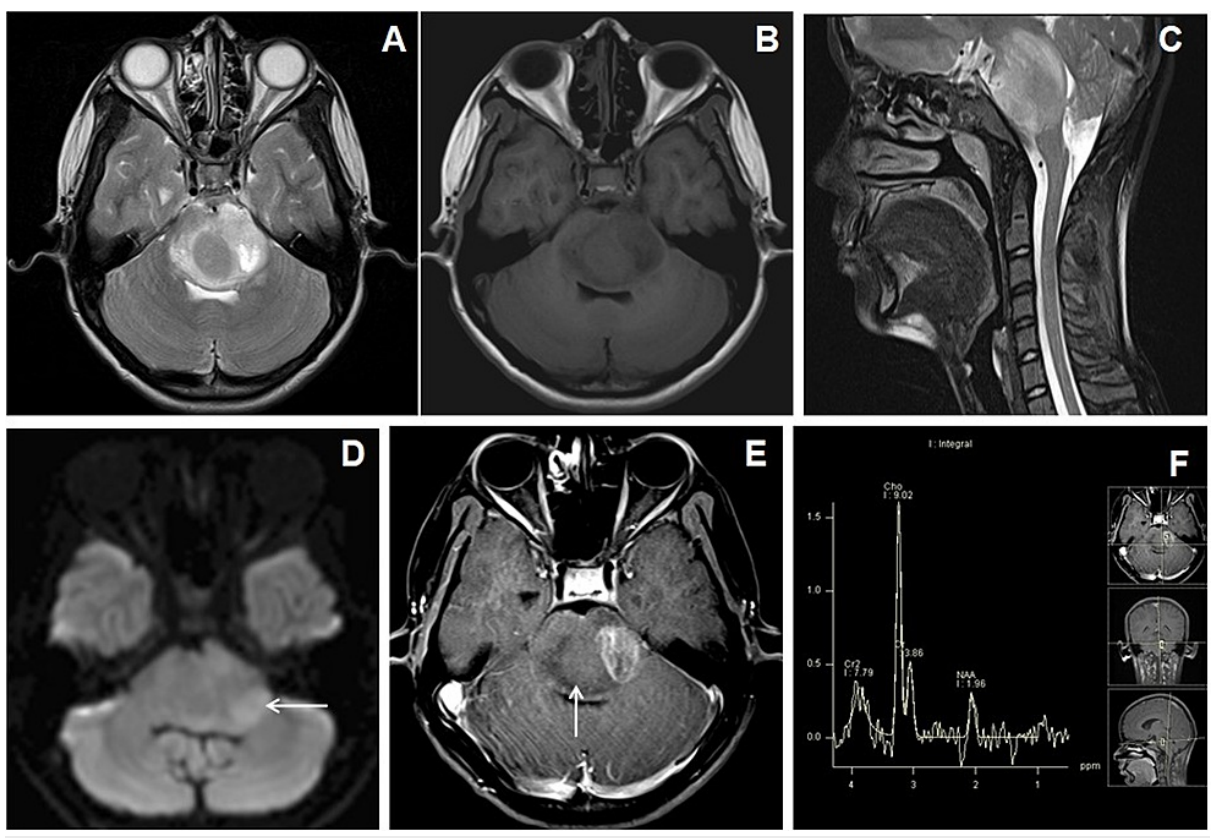

FIGURE 7: Cranial MRI of a 13-year-old female patient with headache and vomiting due to diffuse brainstem glioma.

(A-C) Axial T2WI, T1WI, and sagittal T2WI show central T2W isointense and surrounding T2W hyperintense lesion in the pons, which show central T1W isointensities and peripheral hypointensities. Expansion of the affected brain stem was noted which exerting pressure effect over the fourth ventricle. (D) Axial DWI image shows subtle patchy restriction within the left posterior lateral aspect of the lesion ( $\leftarrow$ arrow). (E) Axial T1W post-contrast image shows patchy heterogeneous enhancement within the left lateral half of the lesion ( $\uparrow$ arrow). (F) MR spectroscopy image at TE-135 ms shows increased choline peak and reduction of NAA peak.

NAA: N-acetyl aspartate; DWI: diffusion-weighted imaging

Of 26 posterior fossa tumors, 18 (45\%) were malignant and eight (20\%) were benign tumors. The mean ADC value of benign tumors was $1.279 \pm 0.38 \times 10^{-3} \mathrm{~mm}^{2} / \mathrm{s}$ and malignant tumors was $0.954 \pm 0.27 \times 10-{ }^{3} \mathrm{~mm}^{2} / \mathrm{s}$ with a statistical significance of $\mathrm{p}=0.019$. The cut-off mean ADC value was $1.022 \times 10^{-3} \mathrm{~mm}^{2} / \mathrm{s}$ with a sensitivity of $78.6 \%$ and specificity of $66.7 \%$ (Figure 8 ). 


\section{Cureus}

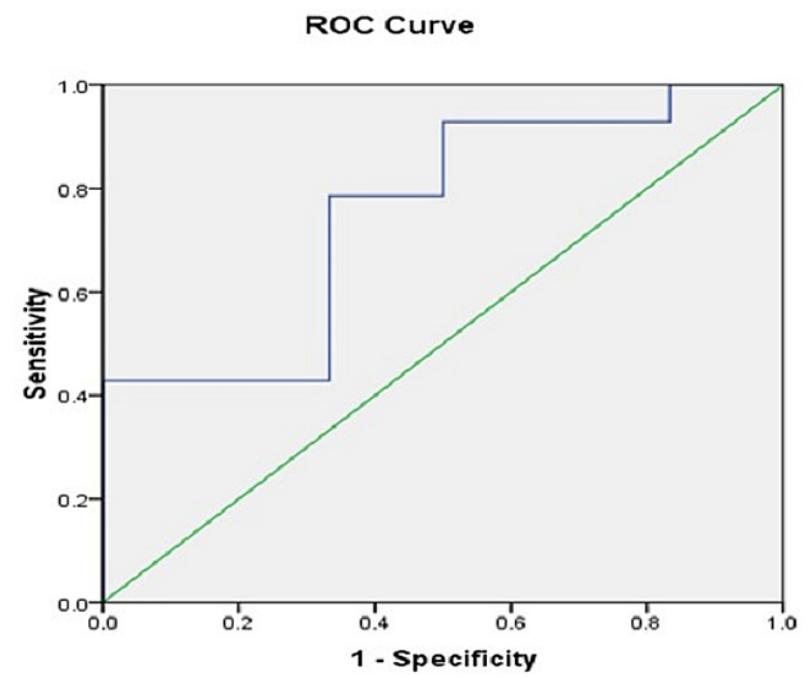

\begin{tabular}{|l|l|l|}
\hline Criteria (Cut-off ADC value) & Sensitivity (\%) & Specificity (\%) \\
\hline$\leq 0.987$ & 71.4 & 66.7 \\
\hline$\leq 1.022$ & 78.6 & 66.7 \\
\hline$\leq 1.252$ & 85.7 & 50 \\
\hline
\end{tabular}

FIGURE 8: ROC curve analysis for mean ADC values of benign and malignant posterior fossa tumors in 26 patients.

ROC: receiver operating characteristic; ADC: apparent diffusion coefficient

The mean Cho/cr ratio for benign and malignant posterior fossa tumors were $1.01 \pm 0.24$ and $1.96 \pm 0.42$ respectively with a statistically significant difference $(\mathrm{p}=0.0005)$. The cut-off $\mathrm{Cho} / \mathrm{cr}$ ratio was 1.25 with a sensitivity of $66.7 \%$, specificity of $85.7 \%$ (Figure 9 ). 
ROC Curve

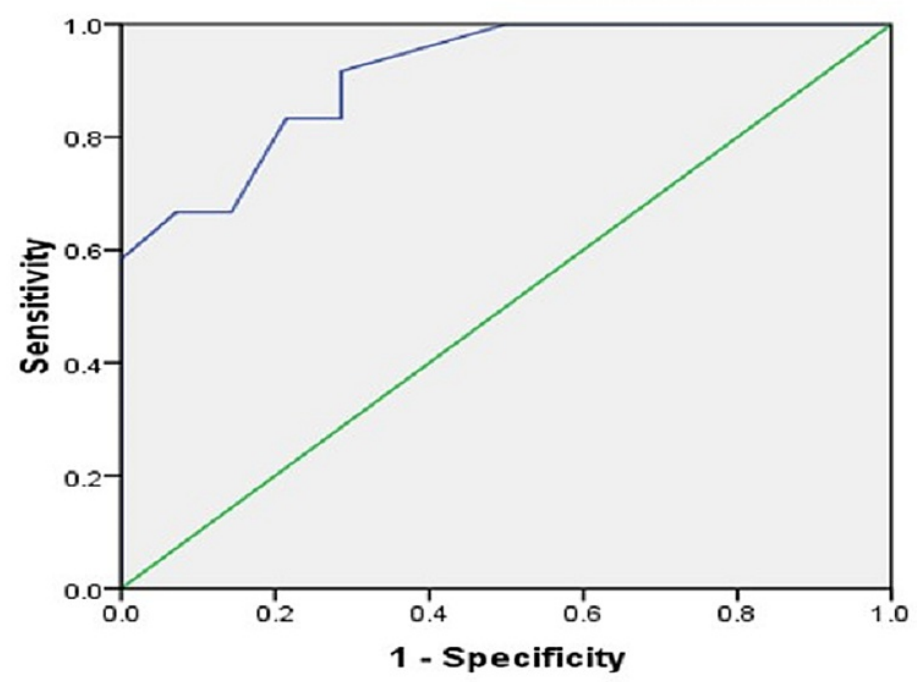

\begin{tabular}{|l|l|l|}
\hline Criteria (Cut-off ADC value) & Sensitivity (\%) & Specificity (\%) \\
\hline$\leq 1.150$ & 66.7 & 92.9 \\
\hline$\leq 1.250$ & 66.7 & 85.7 \\
\hline$\leq 1.350$ & 83.3 & 78.6 \\
\hline
\end{tabular}

\section{FIGURE 9: ROC curve analysis for Cho/cr ratio in benign and malignant posterior fossa tumors in 26 patients.}

ROC: receiver operating characteristic; Cho/cr ratio: choline/creatinine ratio

\section{Discussion}

Forty cases of various posterior fossa space-occupying lesions were evaluated in this study. Tumors, infective lesions, cystic lesions, and tumor-like lesions were considered space-occupying lesions of the posterior fossa.

Metastases were the most common intra-axial lesion to affect the posterior fossa in our study followed by brainstem glioma. In our study, metastatic lesions showed reduced NAA level, elevated choline peak, Cho/cr ratio, and prominent lipid-lactate peak which correlated with the study conducted by Ningapa et al. [8] and Meyer et al. [9]. No elevated choline peak was observed in perilesional edema. High-grade glioma shows similar MR spectral findings, however, there were increased choline peak, Cho/NAA, and Cho/cr ratios in perilesional edema and correlated with previous studies by Law et al. [10], Wang et al. [11], Tsougos et al. [12]. The brainstem gliomas and medulloblastoma showed areas of diffusion restriction with low ADC values along with a raise choline peak and Cho/cr ratio in our study sample [13-16]. Pilocytic astrocytoma and hemangioblastoma did not show diffusion restriction [17].

Vestibular schwannomas diagnosed on basis of its classical location at the opening of the internal auditory canal, extension along the course of the eighth cranial nerve and classic "ice-cream on cone appearance" diagnosed on conventional MR images [18].

Diffusion-weighted imaging with ADC values plays an important role in differentiating arachnoid cyst from an epidermoid cyst in with epidermoid cysts owing to their cellular content exhibiting diffuse restriction with lower to variable ADC values $[4,19]$.

In our study, MR spectroscopy showed prominent amino acid and succinate peaks in pyogenic abscesses, prominent lipid peaks in a tubercular abscess. These MR spectroscopy findings are correlated with previous studies of Karatag and Karatag [20], Gupta et al. [21], Kumar et al. [22], and Meena et al. [23].

Kumar et al. found a good correlation of advanced MRI imaging in the evaluation of posterior fossa tumors with histopathological diagnosis [2]. Darweesh et al. and Moller-Hartmann et al. found a more added value of a combination of MR spectroscopy and ADC values over conventional MR sequences in the differentiation and grading of brain tumors [24,25]. In our study, we found similar observations wherein the use of DWI and 
MRS increased the diagnostic accuracy of MRI in posterior fossa lesions.

\section{Limitation}

The major limitations of our study were the heterogeneous nature of the different space-occupying lesions of the posterior fossa we included and the fewer number of non-neoplastic lesions we encountered in our study sample.

\section{Conclusions}

MRI is the imaging modality of choice for better characterization and differentiation of various spaceoccupying lesions of posterior fossa lesions because of its superior soft-tissue resolution and multiplanar capabilities. Adding DWI and MR spectroscopy over conventional MRI sequences helps us make the diagnosis much closer to the histopathological diagnosis.

\section{Additional Information}

\section{Disclosures}

Human subjects: Consent was obtained or waived by all participants in this study. Assam Medical College Institutional Ethics Committee (H) issued approval No. AMC/EC/PG 2153. Animal subjects: All authors have confirmed that this study did not involve animal subjects or tissue. Conflicts of interest: In compliance with the ICMJE uniform disclosure form, all authors declare the following: Payment/services info: All authors have declared that no financial support was received from any organization for the submitted work. Financial relationships: All authors have declared that they have no financial relationships at present or within the previous three years with any organizations that might have an interest in the submitted work. Other relationships: All authors have declared that there are no other relationships or activities that could appear to have influenced the submitted work.

\section{References}

1. Rai R, Iwanaga J, Shokouhi G, Oskouian RJ, Tubbs RS: The tentorium cerebelli: a comprehensive review including its anatomy, embryology, and surgical techniques. Cureus. 2018, 10:e3079. 10.7759/cureus.3079

2. Kumar TR, Kalra VB, Pakhale HP, Toppo JN, Chhatria M: Role of MRI in common posterior fossa tumors . J Evid Based Med Healthc. 2015, 2:41-47.

3. Cruz Jr LCH, Gasparetto EL, Domingues RC, Domingues RC: Diffusion-weighted MR imaging in brain tumor . Clin Neurol. 2008, 2:21-29.

4. Baghdady AI, Maaly MA, El-wakee AM, Mousa WA: The role of diffusion-weighted MRI in the evaluation and differentiation of space-occupying brain lesions. Menoufia Med J. 2016, 29:303-311. 10.4103/11102098.192445

5. Bulakbasi N, Kocaoglu M, Örs F, Tayfun C, Üçöz T: Combination of single-voxel proton MR spectroscopy and apparent diffusion coefficient calculation in the evaluation of common brain tumors. AJNR Am J Neuroradiol. 2003, 24:225-233.

6. Haaga JR, Lanzieri CF: CT and MRI Anatomy of the Whole Body (Fourth Edition) . Mosby, Inc., Ohio, USA; 2003.

7. Alam MS, Sajjad Z, Hafeez S, Akhter W: Magnetic resonance spectroscopy in focal brain lesions . J Pak Med Assoc. 2011, 61:540-543.

8. Ningappa R, Singh KB, Uppinal SP, Nagaraj BR: The role of diffusion-weighted magnetic resonance imaging in the evaluation of brain mass lesions. West Afr J Radiol. 2016, 23:76-81. 10.4103/1115-1474.164875

9. Meyer HJ, Fiedler E, Kornhuber M, Spielmann RP, Surov A: Comparison of diffusion-weighted imaging findings in brain metastases of different origin. Clin Imaging. 2015, 39:965-969. 10.1016/j.clinimag.2015.06.015

10. Law M, Cha S, Knopp EA, Johnson G, Arnett J, Litt AW: High-grade gliomas and solitary metastases: differentiation by using perfusion and proton spectroscopic MR imaging. Radiology. 2002, 222:715-721. 10.1148/radiol.2223010558

11. Wang Q, Zhang J, Xu W, Chen X, Zhang J, Xu B: Role of magnetic resonance spectroscopy to differentiate high-grade gliomas from metastases. Tumour Biol. 2017, 39:10.1177/1010428317710030

12. Tsougos I, Svolos P, Kousi E, Fountas K, Theodorou K, Fezoulidis I, Kapsalaki E: Differentiation of glioblastoma multiforme from metastatic brain tumor using proton magnetic resonance spectroscopy, diffusion and perfusion metrics at 3 T. Cancer Imaging. 2012, 12:423-436. 10.1102/1470-7330.2012.0038

13. Hari PS, Jyothi JS, Thatipamula M: Study of posterior fossa tumors by high resolution MRI . J Evid Based Med Healthc. 2016, 3:197-203. 10.18410/jebmh/2016/46

14. Poretti A, Meoded A, Huisman TA: Neuroimaging of pediatric posterior fossa tumors including review of the literature. J Magn Reson Imaging. 2012, 35:32-47. 10.1002/jmri.22722

15. Plaza MJ, Borja MJ, Altman N, Saigal G: Conventional and advanced MRI features of pediatric intracranial tumors: posterior fossa and suprasellar tumors. AJR Am J Roentgenol. 2013, 200:1115-1124. 10.2214/AJR.12.9725

16. O’Brien WT Sr: Imaging of primary posterior fossa brain tumors in children . J Am Osteopath Coll Radiol. 2013, 2:2-12.

17. Jaremko JL, Jans LB, Coleman LT, Ditchfield MR: Value and limitations of diffusion-weighted imaging in grading and diagnosis of pediatric posterior fossa tumors. AJNR Am J Neuroradiol. 2010, 31:1613-1616. 10.3174/ajnr.A2155

18. Posterior fossa tumors in adults: MR imaging . (2017). https://epos.myesr.org/poster/esr/ecr2017/C-2241 


\section{Cureus}

19. Diffusion weighted imaging in non ischemic brain disorders . (2013). https://epos.myesr.org/poster/esr/ecr2013/C-2348.

20. Karatag O, Karatag GY, Uysal E, Meltem CS, Mehmet E, Muzaffer B: Can magnetic resonance spectroscopy adequately differentiate neoplastic from non-neoplastic and low-grade from high-grade lesions in brain masses?. Marmara Med J. 2010, 23:326-338.

21. Gupta RK, Jobanputra KJ, Yadav A: MR spectroscopy in brain infections . Neuroimaging Clin N Am. 2013, 23:475-498. 10.1016/j.nic.2013.03.004

22. Kumar A, Kaushik S, Tripathi RP, Kaur P, Khushu S: Role of in vivo proton MR spectroscopy in the evaluation of adult brain lesions: our preliminary experience. Neurol India. 2003, 51:474-478.

23. Diagnostic role of magnetic resonance spectroscopy in intracranial space occupying lesions (IC-SOLs): a prospective analytical study. (2015). https://epos.myesr.org/poster/esr/ecr2015/C-0475.

24. Darweesh AMN, Badawy ME, Hamesa M, Saber N: Magnetic resonance spectroscopy and diffusion imaging in the evaluation of neoplastic brain lesions. Egypt J Radiol Nucl Med. 2014, 45:485-493. 10.1016/i.ejrnm.2014.03.002

25. Möller-Hartmann W, Herminghaus S, Krings T, Marquardt G, Lanfermann H, Pilatus U, Zanella FE: Clinical application of proton magnetic resonance spectroscopy in the diagnosis of intracranial mass lesions. Neuroradiology. 2002, 44:371-381. 10.1007/s00234-001-0760-0 